\title{
Standardizing the Assessment of the Clinical Abilities of Physical Therapists and Occupational Therapists Using OSCE
}

\author{
Yoshikiyo Kanada, RPT, PhD ${ }^{1,2)}$, Hiroaki SAKurai, RPT, $\mathrm{PhD}^{1,2)}$, \\ Yoshito Sugiura, RPT, MS ${ }^{3)}$, Ikuo Motoya, RPT, MS ${ }^{4)}$, Masao Tomita, RPT ${ }^{1,2)}$, \\ Syunji Sawa, OTR, PhD ${ }^{1,2)}$, Toshio Teranishi, RPT, PhD ${ }^{1,2)}$, Tetsuo Okanisi, RPT, PhD ${ }^{1,2)}$ \\ 1) Fujita Health University School of Health Sciences \\ 2) Fujita Health University Graduate School of Health Sciences \\ 3) Department of Rehabilitation, Health Care Service Facility for the Aged, Tobahouwaen \\ 4) Kawamura Hospital: 1-98 Dengakugakubo, Kutsukake, Toyoake, Aichi 470-1192, Japan. \\ TEL: +81 562-93-9000,FAX: +81 562-93-6817,E-mail:yokanada@fujita-hu.ac.jp
}

\begin{abstract}
Purpose] The objective of this study was to clarify the relationship between the results of the Objective Structured Clinical Examination (OSCE) and academic and clinical training achievements, which may be significant in terms of the establishment of standards for the evaluation of therapists' clinical performance. [Subjects] The study surveyed 46 physical and 43 occupational therapists who enrolled at our university in April 2007 and graduated from it in March 2011. [Methods] The OSCE results and academic and clinical training achievements were scored to examine the relationship between them by calculating Spearman's rank correlation coefficients. [Results] A significant correlation was observed between academic achievements and between clinical training achievements, while the correlation between the OSCE results was shown to be high or low. The correlation between the OSCE results and academic achievements was significant until the third grade (OSCE Level 2) and non-significant in the fourth grade (OSCE Level 3) requiring application. The correlation between the OSCE results and clinical training achievements showed a tendency to be low. [Conclusion] These results suggest the necessity of reconsidering the adoption of an OSCE system focusing on consistency between the contents of clinical training and academic education.

Key words: OSCE, Clinical abilities, Standardizing
\end{abstract}

(This article was submitted Apr. 27, 2012, and was accepted May 29, 2012)

\section{INTRODUCTION}

In physical therapy and occupational therapy, the two major branches of rehabilitation medicine, physical therapists and occupational therapists (hereafter referred to as "therapists") administer therapeutic benefits by physically exercising, manipulating the movements of, and providing guidance to patients. Most directions given by physicians in the field of rehabilitation medicine include therapy and training conducted by therapists. Therapists create a therapy program based on a physician's instructions and then carry out the therapy and training. Therefore, therapists play an important role that directly affects patients. In other words, the clinical ability (knowledge, skills, professional attitude) of therapists is the largest deciding factor of the therapeutic result. However, there was a time when physical therapy schools were established one after another in an unsystematic way, and this has resulted in disparities in the clinical abilities of therapists, which has led to clear differences in therapeutic results ${ }^{1)}$.

The increasing demand for therapists from 1990 to 2000 brought about by the advent of Japan's aging society caused a sudden increase in the establishment of schools for therapists (hereafter referred to as "therapy schools"). This has resulted not only in disparity among the schools but also in a lowering of the quality of therapists who are educated at these schools. This has in turn led to a lowering of the quality of rehabilitation medicine therapy being offered at medical facilities, which is a major problem in the medical system ${ }^{1)}$.

In 2000 both the effort to make therapy education part of university curriculums and the influence of the declining birth rate in Japan resulted in therapy schools that had formerly been run as vocational schools suddenly being unprofitable due to under enrollment. These schools also began to worry about the lowering of the national examination pass rate, the reduction in the number of places of employment, and how to deal with the inclusion of their educational programs in university curriculums. As a result, the current levels of knowledge, skills, and the professional attitude of therapy school students are coming into question.

One result of this is the fact that many young therapists become members of teams at their workplaces without having had clinical training, and often work for many years unaware that they need to improve their knowledge and 
skills. This has in turn led to a deterioration in the quality of the clinical training provided to students. Measures to deal with as well as a solution to the problem of the decreasing quality of pre- and post-graduation education and training must be found as soon as possible.

We consider physical and occupational therapy to be "the study of treatment, " which is to say a clinical science, and we identify student education in this field as a professional specialized education, or put another way, the education to produce specialists who have clinical skills. In other words, the main task of this type of education is clinical skill training. In the current therapist education system, an instructor does not assess the students' clinical abilities. Rather, the assessment is performed by the mentor of the facility where the students do their clinical training. In a therapist education system designed to produce clinicians, this is an issue of the utmost importance. Our instructors regularly treat patients in clinical settings and hold regular discussions with therapists working there on how students should be trained. At the university, we are also working to clarify standards for the clinical education that takes place in therapy schools, to using a version of the OSCE (Objective Structured Clinical Examination) tailored to therapists, and could standardize the assessments carried out by clinical training mentors ${ }^{2}$. We believe that if we establish standardization of both treatment and assessment, this would lead to improvement in the quality of rehabilitation medicine facilities.

Thus, we introduced OSCE in all courses in 2005 and an OSCE seminar as a course in 2011. In addition, we had the clinical training mentors become familiar with the details of OSCE in this field in an attempting to standardize student assessment. Through these means we aspire to use OSCE as a way to bridge the gaps between classroom education and education via clinical training, and integrate the education given by therapy schools and with that given by clinical training facilities, which is something therapy educational institutions throughout Japan have so far been unable to do.

The present study is significant in terms of establishing standards for the assessment of the clinical abilities of therapists. Its objective is to elucidate the relationship between OSCE performance, classroom performance, and clinical training performance.

\section{SUBJECTS AND METHODS}

The subjects were 46 students of physical therapy and 43 students of occupational therapy, they were all of whom were enrolled at our university in April 2007 and graduated in March 2011.

At our university, instructional modes are under constant review with the aim of the level of academic achievement and the retention rate of education.

Training (clinical training) conducted at clinical sites and the OSCE employed at our university include early experiential training (2nd year), clinical training (3rd year), and applied clinical training (4th year). The early experience training ( 3 weeks in duration) is intended to provide basic knowledge, skills, and foster a professional attitude (with particular emphasis on professional etiquette), as well as certain elements of basic assessment techniques. It is designed so that students achieve the following: mastery of the skills required to carry out OSCE Level 1 and gain experience in OSCE Level 2 . The clinical training (6 weeks each at 3 facilities) ensures the students learn the basics of assessment and the process of treatment as well as learn the knowledge and skills required to become independent physical and occupational therapists, and is designed to enable so that students to achieve mastery of the skills required to carry out OSCE Levels $1,2,3 \mathrm{a}$, and $3 \mathrm{~b}$. The applied clinical training (6 to 7 weeks each at 2 facilities) provides students with clinical training at facilities outside the university and is designed to provide them with the chance to learn and experience the practical assessment techniques and treatment processes required of physical and occupational therapists to enable them to mastery of the skills required to carry out OSCE Levels 1, 2, 3a, 3b, and 3c. Scoring is broadly divided into "Basic professional attitude as a medical professional," "assessment," and "treatment." Early experiential training has 23 scoring categories, and clinical training and applied clinical training each have 40 scoring categories. Scores are expressed in 6 levels ranging from "not implemented for scoring," which is 0 points, to "graduate (working as a novice) level," which is 5 points.

OSCE categories are shown in Table 1. Level 1 (assessment before and after early experiential training) provides mastery of communication skills and nursing care techniques. The categories are standard preventative measures, communication skills, hot packs, triangular bandages (upper extremity management), fitting patients with orthoses, assisted wheelchair operation, and assisted transfer (2-person assisted transfer). Level 2 (assessment before and after clinical training) provides mastery of testing and measuring skills. The categories are interviewing therapists, vital signs check, tendon reflex testing, pathological reflex testing, joint range of motion measurement, manual muscle testing, sensory testing, and morphometry. Level 3 (assessment before and after applied clinical training) provides mastery of analytical and treatment techniques (assistance and guidance), including sitting up in bed, standing up, transferring, getting dressed, using the toilet, and walking. Level 3 is further divided into: a) motion analysis, and treatments for $\mathrm{b}$ ) patients assessed as equivalent to FIM3, and c) severely ill patients assessed as equivalent to FIM2. Implementation methods for OSCE include preparing several rooms ("stations") and holding examinations for each category in separate rooms. The test takers (students) rotate through the stations according to the directions of the test administrators and are examined in each category of the OSCE. There are two evaluators and 1 mock patient at each station. These roles are played by physical and occupational therapists who are also instructors. Students spend 5 minutes at each station and receive 2 minutes of feedback after they have completed each category. The OSCE test categories that are to be tested are not previously announced to the test takers.

Statistical processing was done using Predictive Analytics Software (PASW) Statistics 18.0. To compare 
Table 1. OSCE categories

\begin{tabular}{ll}
\hline Level 1 & communication skills and nursing care techniques \\
& standard preventative measures, communication skills, hot packs, \\
& triangular bandages (upper extremity management), \\
& fitting patients with orthoses, assisted wheelchair operation, \\
& assisted transfer (2-person assisted transfer) \\
\hline Level 2 & provides mastery of testing and measuring skills \\
& interviewing therapists, vital signs check, tendon reflex testing, \\
& pathological reflex testing, joint range of motion measurement, manual muscle testing, \\
& sensory testing, morphometry \\
\hline Level 3 & provides mastery of analytical and treatment techniques (assistance and guidance) \\
& getting up in bed, standing up, transferring, getting dressed, using the toilet, walking \\
& a:motion analysis \\
& b\&c:treatment (b:patient assignment equivalent to FIM3, \\
& c: severely ill patient assignment equivalent to FIM2) \\
\hline
\end{tabular}

among more than three results at each period of clinical training (first, second, third period of junior and first, second period of senior), one-way ANOVA and TukeyKramer's multiple comparison were used. The score ratios for OSCE performance, classroom performance, and clinical training performance were calculated and the relationship between them was investigated using Spearman's rank order correlation coefficent. Because the concordance rate of 2 evaluators can be low, the two evaluators chose one score from among the performance scores that they had discussed the one to use as the OSCE performance score. The classroom performance score was the average of the scores of each year.

\section{RESULTS}

Clinical training performance showed significant improvement between the first and second terms of the 3rd year, between the second and third terms of the 3rd year, and between the third term of the 3rd year and the first term of the 4 th year.

The relationships between OSCE performance, classroom performance, and clinical training performance are shown in Table 2. There was a correlation between performance in 1st, 2nd, and 3rd year classroom lectures. Some OSCE performance scores were grouped highly and some were grouped at lower levels. Clinical training performance scores were weakly correlated. The relationship between classroom and OSCE performance showed significant correlation until the 3rd year (OSCE Levels 1 and 2), but there was no correlation between classroom performance and post-applied clinical training Level 3. In terms of the relationship between classroom and clinical training performance, there was a significant relationship between initial experiential training and clinical training, but these showed no relationship to applied clinical training. There was a correlation between OSCE and clinical training groups at lower levels.

\section{DISCUSSION}

Rehabilitation medicine is a practical system that applies learning to medical treatment. Thus, those of us who are proud to be specialists in education prefer not to simply transfer the structure created at medical departments to therapy education, but would rather search for a new significance of OSCE from the viewpoint of rehabilitation medicine and create a new structure based on the new information we are able to obtain. In the present study, we have described how we have used OSCE to bridge the gaps between classroom education and clinical training education programs and will use this to establish clinical education assessment standards for therapists as the first step toward our the education given by schools with that given by training facilities. To that end, this study elucidated the correlation between OSCE performance, classroom performance, and clinical training performance.

The average score for clinical training performance improved with time line. Previously, at our university, we had set training achievement levels for each clinical training term. However, using this method often caused regional disparities in the achievement levels and disparities in the guidance methods used by training mentors, which in turn caused disparities in the level of difficulty of the training. This created confusion in the students' studies and many students became confused about how they should study. Therefore, we believe that the improvement in clinical training performance along with time is the result of clarifying the points at which achievement is reached for both the students and the mentors, by the introduction of uniform making the assessment standards we use from the first clinical training to the final clinical training application, uniform and by having mentors at clinical training facilities become familiar with and use a training assessment chart.

There was a strong correlation between the 3 categories in the $1 \mathrm{st}, 2 \mathrm{nd}$, and $3 \mathrm{rd}$ years of classroom performance. Yoshizawa et al. have studied the contribution the motivation to learn makes to the improvement of classroom performance ${ }^{3)}$. This study did not include a survey of the 


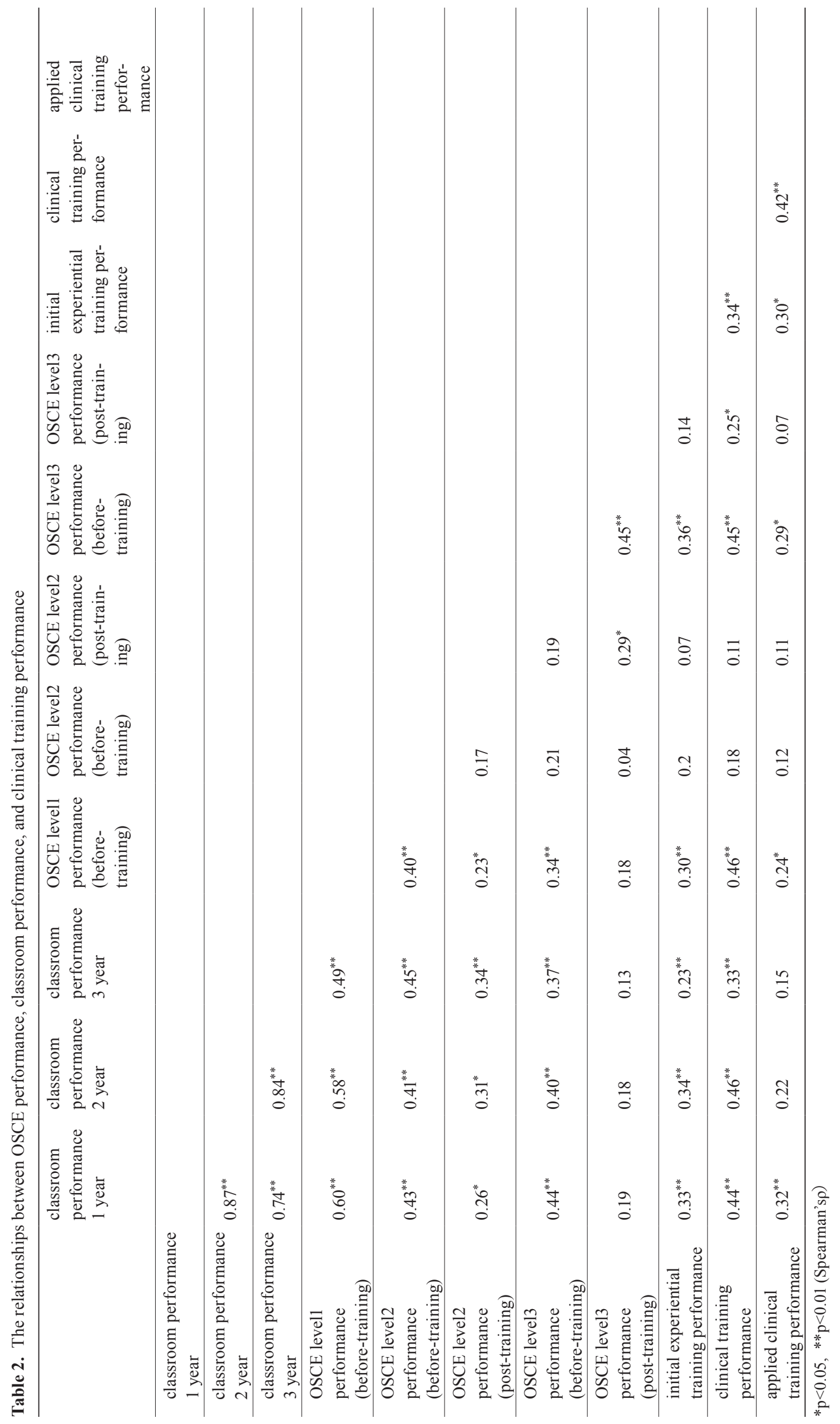


motivation to learn, but 1st year students who scored high in classroom performance had high classroom performance scores in their 2nd and 3rd years as well. However, the fact that the correlation with classroom performance of upperclassmen weakened as the level of OSCE difficulty increased suggests that the correlation between the accumulation of knowledge and the ability to apply that knowledge, the skills the student has, and the student's professional attitude, is weak. Therefore, we believe it is possible to perform a comprehensive assessment of students' clinical abilities using OSCE, which is a simulation test that makes it possible to judge whether a student has achieved the level of ability necessary for clinical training ${ }^{4}$. We therefore believe it is important to introduce OSCE before training begins as a way to both verify the students' knowledge and ability to apply that knowledge.

There was mid-level correlation between classroom performance in the 1st and 2nd years and OSCE Level 1. As the level of difficulty increased from OSCE Level 1 to Level 3, the correlation between OSCE and classroom performance weakened. This suggests that because the details of each OSCE level shift from classroom education, based on knowledge of assisting techniques, tests, etc, to treatment methods that place importance on the emotional domain of dealing with society and patients, abilities other than academic abilities are necessary in a clinical setting. In addition, many of the problems that mentors single out in PT, OT, and other rehabilitation medicine students are related to this emotional domain, such as poor sociality and basic professional attitude ${ }^{5)}$. We believe that this fact underscores the importance of standardization of both the skills considered necessary for clinical practice and the curriculum students are taught. One potential way of addressing this issue proposal would be to help clinical training mentors understand students better and create a more unified educational program by having them participate in OSCE lectures and tests as mock patients or evaluators. However, Saitoh found that instructors in educational facilities specializing in rehabilitation should be "rehabilitation specialists who simultaneously taught their specialty" rather than "education specialists, " and that students should learn by following the example of instructors whom they regarded as outstanding specialists ${ }^{6}$. Thus, we believe instructors should be putting more of their energy into teaching the treatment techniques and methods of dealing with patients that are necessary in a clinical setting.

There was no correlation between OSCE performance and either clinical training or applied clinical training performance. Sato et al. have observed that when taught in classroom lectures, students are able to pass $\mathrm{OSCE}^{7}$ ). It is a mistake to think that the only preparation necessary for the OSCE is an educational program that provides practice in skills and professional attitude. It is also important to study how to apply the knowledge that is learned in clinical settings. In addition, the purpose of OSCE is not only gaining proficiency in skills and professional attitude, but rather its encourage the seeking of the purpose is to seek for wider and deeper knowledge that is required of medical professionals. The results our present of this study indicate that the correlation between OSCE performance and both clinical training and applied clinical training is low, which in turn means that the assessment of students' clinical training does not reflect their clinical abilities. This therefore suggests that it will be necessary in the future to improve OSCE considering the consistency of what is taught in both clinical training and classroom lectures. In comparison with before training was begun, OSCE performance improved after training. Nakahara et al. have reported that students have a favorable attitude toward OSCE and its assessments, and recognize the advantages of OSCE in clinical settings ${ }^{5}$. This means that proactive adoption into clinical training of the OSCE techniques that have been cultivated in the classroom will likely lead to improved OSCE performance, which is one yardstick of the skills required of physical therapists.

In the future, in order to increase the reliability and validity of OSCE assessments, it will be necessary to verify inter-rater reliability and improve the assessment sheet. We would also like to see advances made not only in the education provided to students and novice therapists but also in the effort to standardize the clinical skills taught to students and novice therapists by experienced physical and occupational therapists.

\section{REFERENCES}

1) Kanada Y: A Discussion on Educational Program of Physical Therapist and Occupational Therapist for Effective Rehabilitation. Reprinted from the Fujita Medical Society, 2002, 243-267.

2) Saitoh E, Kanada Y, Tomita M, et al.: The Objective Structured Clinical Examination (OSCE) for Physical Therapist and Occupational Therapist. Tokyo: Kanahara publication, 2011, p275.

3) Yoshizawa T, Ota N, Fujisawa S: Effect of willingness to learn on regular examination results. Rigakuryoho Kagaku, 2008, 23: 249-253. [CrossRef]

4) Miller GE: The assessment of clinical skills/competence/performance. Acad Med, 1990, 65: 63-73. [Medline] [CrossRef]

5) Nakahara M, Murakami S, Morita M, et al.: Transition of student's affective domain and stress during clinical practice. Yanagawariha Fukuokakokusai Kiyo, 2007, 3: 52-56.

6) Saitoh E: A problem of the rehabilitation medical education. Jpn J Rehabil Med, 2005, 42: 388-389.

7) Sato E, Yoshitomi H: Of the business training learning, actually. Pharmaceutical Affairs, 2010, 52: 29-40. 